\title{
Eradication of enterococci biofilms by lactic acid alone and combined with chlorhexidine and cetrimide
}

\author{
María-Teresa Arias-Moliz ${ }^{1}$, Pilar Baca ${ }^{2}$, Santiago Ordóñez-Becerra ${ }^{3}$, María-Paloma González-Rodríguez ${ }^{3}$, \\ Carmen-María Ferrer-Luque ${ }^{3}$
}

\begin{abstract}
${ }^{1}$ DDS, PhD: Assistant Professor. Department of Microbiology, School of Dentistry. University of Granada, Campus de Cartuja, Colegio Máximo s/n, Granada, Spain

${ }^{2}$ DDS, MD, PhD: Professor. Department of Preventive Dentistry, School of Dentistry. University of Granada, Campus de Cartuja, Colegio Máximo s/n, Granada, Spain

${ }^{3}$ BDS: Postgraduate Student, DDS, PhD: Associate Professor, DDS, MD, PhD: Associate Professor. Department of Operative Dentistry, School of Dentistry. University of Granada, Campus de Cartuja, Colegio Máximo s/n, Granada, Spain
\end{abstract}

Correspondence:

Department of Microbiology, School of Dentistry

Campus de Cartuja, Colegio Máximo s/n

E-18071, Granada (Spain)

mtarias@ugr.es

Arias-Moliz MT, Baca P, Ordóñez-Becerra S, González-Rodríguez MP, Ferrer-Luque CM. Eradication of enterococci biofilms by lactic acid alone and combined with chlorhexidine and cetrimide. Med Oral Patol Oral Cir Bucal. 2012 Sep 1;17 (5):e902-6.

http://www.medicinaoral.com/medoralfree01/v17i5/medoralv17i5p902.pdf

Received: 09/12/2011

Accepted: 22/03/2012

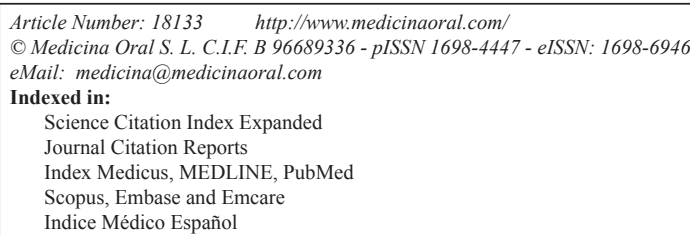

\begin{abstract}
Objective: The antimicrobial activity of lactic acid (LA) alone or in combination with chlorhexidine (CHX) and cetrimide (CTR) against three Enterococcus faecalis strains, E. faecalis ATCC 29212, E. faecalis EF-D1 and E. faecalis U-1765, one Enterococcus durans strain and one dual-species biofilm was investigated.

Study Design: The irrigating solutions tested were 20\%, 15\%, 10\%, 5\% and 2.5\% LA, alone and in combination with $2 \%$ CHX and with $0.2 \%$ CTR. The biofilms were grown in the $\mathrm{MBEC}^{\mathrm{TM}}$ high-throughput device for 24 hours and exposed to the solutions for 30 seconds and 1 minute. "Eradication" was defined as $100 \%$ bacterial kill.

Results: Twenty percent LA eradicated all enterococci biofilms after 30 seconds contact time. The association of LA $+0.2 \%$ CTR achieved better results than LA alone, in contrast with the results obtained using LA $+2 \%$ CHX. E. durans was eradicated by all the tested solutions at 1 minute. The dual-species biofilm, E. faecalis ATCC 29212 $+E$. durans, gave intermediate values of the pure cultures.

Conclusions: LA is capable of eradicating enterococci biofilm at a concentration of $20 \%$. The combination of lower concentrations with $0.2 \%$ CTR achieved eradication after 1 minute.
\end{abstract}

Key words: Biofilm, cetrimide, chlorhexidine, enterococcus durans, enterococcus faecalis. 


\section{Introduction}

The success of root canal treatment depends on the control of microorganisms present in infected root canals (1). Enterococci are commonly isolated bacteria in root canal systems with failed endodontic treatment due to their ability to adhere to dentin and invade dentinal tubules and to form communities organized in biofilms, which may contribute to bacterial resistance and persistence after intracanal antimicrobial procedures (2). The complexity of the root canal system makes complete elimination of bacteria by means of chemo-mechanical treatment nearly impossible (3).

No single irrigant is capable of exerting antimicrobial activity, removing the smear layer, and dissolving organic debris. For this reason, different agents are used in combined form in order to achieve such properties (4). The most widely used antimicrobial agents are sodium hypochlorite and chlorhexidine (CHX), the former outstanding for its ability to dissolve necrotic tissue, and the latter for its substantivity (5). Chelating agents -such as EDTA, citric and maleic acids-have been used in view of their efficacy in eliminating the smear layer $(6,7)$. Maleic acid itself has the capacity to eradicate $E n$ terococcus faecalis biofilms when applied at a clinical concentration of $7 \%$. However, the antimicrobial activity of EDTA and citric acid against biofilms is controversial (8). The combination of the two acids with surfactant agents such as cetrimide (CTR) has nonetheless demonstrated a greater antimicrobial efficacy (9).

The use of lactic acid (LA) as an irrigating solution was introduced by Ayad et al. (10), who showed that it can remove the smear layer, improve the bond strength of the obturation material in contact with the root canal walls $(11,12)$ while also increasing the fracture resistance of thin-walled endodontically treated teeth (13). It is an organic acid that occurs naturally in the muscles during muscular exercise, suggesting it is more biologically acceptable than other chelating agents, and its price makes it economically competitive with endodontic irrigants (11). Due to its nature as an organic acid, it exerts antimicrobial activity against foodborne bacteria (14). However, its action against enterococci strains is unknown, as is its effect when combined with antimicrobial and surfactant agents. The aim of the present study was therefore to evaluate, in vitro, the antimicrobial activity of LA alone or in combination with CHX and CTR against three $E$. faecalis strains, one $E$. durans strain and one dual-species biofilm.

\section{Material and Methods}

\section{Microorganisms and tested materials}

The enterococci strains used in this study and their source were: E. faecalis ATCC 29212, E. faecalis EF-D1 and $E$. durans $\mathrm{ED}-\mathrm{C} 1$ from the collection of the Microbiology Laboratory, School of Dentistry, University of
Granada, obtained from failed endodontic treatment; and E. faecalis U-1765 from a human nosocomial infection, provided by the Microbiology Laboratory, School of Science, University of Granada. Bacterial strains were taken from a $4^{\circ} \mathrm{C}$ stock culture and streaked out twice on BHI (Brain Heart Infusion; Scharlau Chemie S.A., Barcelona, Spain) agar plates for 24 hours at $37^{\circ} \mathrm{C}$. Colonies were suspended in BHI to obtain a 1 McFarland initial bacterial suspension of approximately $3 \times 10^{8}$ colony-forming units per $\mathrm{mL}(\mathrm{CFU} / \mathrm{mL})$. All strain cultures were checked for purity by Gram stain and colony morphology.

The test agents and concentrations assayed were: $20 \%$, $15 \%, 10 \%, 5 \%$ and $2.5 \%$ LA (Panreac, Castellar del Valles, Spain), alone and in combination with $2 \%$ CHX (Guinama, Alboraya, Valencia, Spain) and with $0.2 \%$ CTR (Sigma-Aldrich Chemie, Steinheim, Germany). All the dilutions were carried out using sterile distilled water and were stored at room temperature until use, for no more than 60 minutes.

Biofilm susceptibility test

The biofilm model used in this study was the $\mathrm{MBEC}^{\mathrm{TM}}$ high-throughput (HTP) device (Innovotech, Edmonton, Alberta, Canada) $(8,15)$. To summarize briefly, there are two parts to this batch-culture apparatus. The top half is a lid with 96 pegs that also fits over a standard 96-well microtiter plate. The bottom half is a fluted trough that guides inoculated growth medium across the pegs when the device is placed on a rocker (16). The initial bacterial suspension was diluted 30-fold in BHI broth (Scharlau Chemie S.A., Barcelona, Spain) (approximately $10^{7}$ $\mathrm{CFU} / \mathrm{mL}$ ), and $22 \mathrm{ml}$ of the 1 in 30 dilution was used to inoculate the trough of the MBEC ${ }^{\mathrm{TM}}$-HTP device. Simple biofilms of each of the enterococci strains were created, and one dual-species biofilm was formed through the association of E. faecalis ATCC 29212 and E. durans ED-C1. The peg lid was fitted inside the trough and the assembled device was then placed on a rocking table (OVAN, model Swing Sw 8 10000-00015, Badalona, Spain) and incubated at $37^{\circ} \mathrm{C}$ for 24 hours, at 5 rocks per minute. The shear force of the rocking motion facilitated the formation of 96 equivalent biofilms on the pegs. Biofilms forming on the lid of the $\mathrm{MBEC}^{\mathrm{TM}}-\mathrm{HTP}$ device were rinsed by inserting the peg into a microtiter plate with $0.9 \%$ saline solution per well for 2 minutes. To determine biofilm formation, four pegs were broken off from each device, placed in $200 \mu 10.9 \%$ saline, and sonicated on a water-table sonicator (Branson, model 5510E-MT, Danbury, CT, USA) for 10 minutes (8). The disrupted biofilms were diluted serially and plated for viable cell counting. This growth control was used to evaluate the initial number of bacteria formed in the biofilm in each assay.

The susceptibility tests were done in a microtiter plate known as the 'challenge plate' (Nunclon Delta Surface, 
Nunc, Roskilde, Denmark). The volume of the wells of the challenge plate must be sufficient to submerge the peg past the height of the biofilm produced. We used flat-bottom 96-well microtiter plates (Nunclon Delta Surface; Nunc, Roskilde, Denmark) with a final volume of $200 \mu \mathrm{l}$. The irrigating solutions were placed along the length of the challenge plate, allowing the first and the last wells of each row, containing BHI, to respectively serve as the sterility or negative, and growth or positive controls. The peg lid was submerged in the challenge plate for 30 seconds and 1 minute. Following exposure, the biofilms were rinsed twice as previously described to remove loosely adherent planktonic bacteria, and they were then placed in a microtiter recovery plate with $200 \mu \mathrm{l}$ of BHI per well and sonicated for $10 \mathrm{mi}-$ nutes. Disrupted biofilm cultures were diluted serially and plated for viable cell counting. Five replicates per irrigant concentration, bacteria biofilm and exposure time were performed in 3 different peg lids.

The effect of each test agent on the biofilm was determined by calculating the percentage of viable bacteria kill respect to the control as follows: [1-(mean $\mathrm{CFU}_{\text {irrigant }}$ $\left.\left.\mathrm{CFU}_{\text {initial bacterial number }}\right)\right] \times 100$. The term 'eradication' was used to denote the death of $100 \%$ of the bacterial population.

\section{Results}

The results of the antibacterial effects of varied dilutions of LA and their combinations with CHX and CTR are listed in (Table 1). Twenty percent LA eradicated all enterococci biofilms after 30 seconds contact time. At 1 minute of exposure it eradicated them at a lesser concentration, $15 \%$.

Table 1. Kill percentage of enterococci biofilms by lactic acid (LA) and the combinations of $2 \%$ chlorhexidine (CHX) and $0.2 \%$ cetrimide (CTR) after 30 seconds and 1 minute contact time. Mean (SD).

\begin{tabular}{|c|c|c|c|c|c|c|c|c|c|c|}
\hline & \multicolumn{2}{|c|}{ E. faecalis ATCC 29212} & \multicolumn{2}{|c|}{ E. faecalis EF-D1 } & \multicolumn{2}{|c|}{ E. faecalis $\mathrm{U}-1765$} & \multicolumn{2}{|c|}{ E. durans ED-C1 } & \multicolumn{2}{|c|}{$\begin{array}{c}\text { E. faecalis ATCC } 29212 \\
\text { E. durans ED-C1 }\end{array}$} \\
\hline & 30 seconds & 1 minute & 30 seconds & 1 minute & 30 seconds & 1 minute & 30 seconds & 1 minute & 30 seconds & 1 minute \\
\hline $20 \% \mathrm{LA}$ & E & E & $\mathrm{E}$ & E & E & E & E & E & E & E \\
\hline $15 \% \mathrm{LA}$ & E & E & $90.81(10.21)$ & E & $98.50(2.85)$ & E & $99.61(0.58)$ & E & E & E \\
\hline $10 \% \mathrm{LA}$ & $43.11(19.09)$ & $90.17(8.09)$ & $84.91(6.69)$ & $9.14(3.73)$ & $95.33(1.65)$ & $95.57(1.92)$ & $99.29(0.86)$ & E & $97.29(2.45)$ & E \\
\hline $5 \%$ LA & $12.88(7.09)$ & $67.01(5.78)$ & $35.08(11.68)$ & $40.74(4.83)$ & $76.34(3.82)$ & $90.21(5.09)$ & $98.19(2.86)$ & $\mathrm{E}$ & $69.09(2.11)$ & $99.77(0.34)$ \\
\hline $2.5 \% \mathrm{LA}$ & $4.88(27.14)$ & $9.47(12.87)$ & $14.09(32.40)$ & $28.88(32.02)$ & $61.15(5.67)$ & $71.16(10.42)$ & $89.37(2.53)$ & E & $24.93(27.77)$ & $95.69(7.18)$ \\
\hline $20 \% \mathrm{LA}+2 \% \mathrm{CHX}$ & $96(2.89)$ & E & E & E & $99.96(0.086)$ & E & $\mathrm{E}$ & E & E & E \\
\hline $15 \% \mathrm{LA}+2 \% \mathrm{CHX}$ & $92(2.53)$ & E & $80.32(6.48)$ & $96.20(2.05)$ & $95.34(1.14)$ & $97.90(1.08)$ & $96.32(3.55)$ & E & $97.94(0.83)$ & E \\
\hline $10 \% \mathrm{LA}+2 \% \mathrm{CHX}$ & $75.11(4.27)$ & $72.28(8.29)$ & $58.03(6.3)$ & $57.42(11.73)$ & $89.53(1.26)$ & $95.34(1.58)$ & $96.53(1.91)$ & E & $96.58(3.29)$ & $99.93(0.1)$ \\
\hline $5 \% \mathrm{LA}+2 \%$ CHX & $45.53(11.39)$ & $47.71(9.59)$ & $21.96(21.42)$ & $26.37(41.32)$ & $81.92(3.55)$ & $82(7.56)$ & $87.25(6.45)$ & E & $74.08(16.43)$ & $99.66(0.17)$ \\
\hline $2.5 \% \mathrm{LA}+2 \% \mathrm{CHX}$ & $12.44(15.99)$ & $16.14(27.72)$ & $12.78(39.99)$ & $4.59(56.36)$ & $53.65(8.17)$ & $62.07(5.56)$ & $28.12(40.74)$ & E & $32.05(28.82)$ & $51.51(44.92)$ \\
\hline $20 \% \mathrm{LA}+0.2 \% \mathrm{CTR}$ & E & E & E & E & E & E & E & E & E & E \\
\hline $15 \%$ LA $+0.2 \%$ CTR & E & E & $\mathrm{E}$ & E & E & E & E & E & E & E \\
\hline $10 \% \mathrm{LA}+0.2 \% \mathrm{CTR}$ & E & E & $99.34(1.46)$ & E & E & E & E & E & E & E \\
\hline $5 \% \mathrm{LA}+0.2 \% \mathrm{CTR}$ & E & $\mathrm{E}$ & $94.09(5.38)$ & E & E & E & $99.64(0.32)$ & E & $99.78(0.24)$ & E \\
\hline $2.5 \% \mathrm{LA}+0.2 \% \mathrm{CTR}$ & E & $\mathrm{E}$ & $88.19(7.89)$ & E & E & E & $99.10(0.46)$ & E & $69.04(22.91)$ & $\mathrm{E}$ \\
\hline
\end{tabular}

E: eradication (100\% kill).

SD: standard deviation 
Globally, the association of LA $+0.2 \%$ CTR achieved better results than LA alone, in contrast with the results obtained using LA $+2 \% \mathrm{CHX}$. The combinations of all the concentrations of LA $+0.2 \%$ CTR eradicated the biofilms of E. faecalis ATCC 29212 and U-1765 at 1 minute and 30 seconds of contact time. The biofilms of the other two strains and the dual-species biofilm experimented $100 \%$ kill, but only at 1 minute.

E. durans was very sensitive to exposure times of 1 minute, and was eradicated by all the tested solutions (alone and combined). The dual-species biofilm E. faecalis ATCC 29212 with E. durans gave intermediate values of the pure cultures.

\section{Discussion}

The use of chelating agents associated with antimicrobial and surfactant agents has been tested in attempts to remove the smear layer and control microorganisms from the root canal system. The lack of studies that evaluate enterococci biofilm susceptibility to LA chelating solution applied alone, or else in association with antimicrobials and surfactants, led us to explore the biofilm eradication capacity of different concentrations of LA and their combination with $2 \% \mathrm{CHX}$ and $0.2 \%$ CTR.

The method selected was the MBEC ${ }^{\text {TM }}$ HTP, as it has been previously used for the in vitro testing of the antimicrobial activity of irrigants, allowing us to compare the results. In this device, four single and one dual enterococci strain biofilms were grown for 24 hours, all strains except one being E. faecalis, which are often isolated from necrotic or improperly filled root canal systems $(17,18)$. Namely, the strains studied were $E$. faecalis ATCC 29212, a strain of reference widely used in antimicrobial susceptibility studies (19); E. faecalis U-1765 isolated from a nosocomial infection; and two enterococci strains isolated from failed endodontic treatments, E. faecalis EF-D1 and E. durans ED-C1, the latter reportedly present in root canals (20). In addition, a dual-species biofilm was assayed (E. faecalis ATCC $29212+$ E. durans ED-C1) due to the fact that endodontic infections are typically polymicrobial (21).

LA was selected as it has been previously reported to effectively remove the smear layer from root canal (10) and to enhance the bond strength of Epiphany adhesive sealer to human dentin at 10 and $20 \%$ (11). In this study, the biofilms were eliminated by $20 \%$ LA after both contact times, and by a concentration of $15 \%$ after 1 minute. The antimicrobial activity of LA may stem from its chemical nature as an organic acid. Organic acids decrease the internal $\mathrm{pH}$ of microbial cell through the ionization of dissociated acid molecules, and the disruption of substrate transport by altering cell membrane permeability (14). The low molecular weight of LA (90.08 Dalton) lead to smaller nondissociated mo-lecules that could enter the bacterial cells easily, and change the internal $\mathrm{pH}$ of the organisms.
LA was combined with $0.2 \% \mathrm{CTR}$, a cationic surfactant that reduces the surface tension of liquids (22). It has shown ability to eradicate E. faecalis biofilm in vitro (23) and, in human dentin, its antimicrobial residual activity persisting over time (24). In this study, all combinations eradicated the enterococci biofilms after 1 minute and the biofilms of E. faecalis ATCC 29212 and E. faecalis U-1765 after 30 seconds. These findings indicate that this association enhances both solutions' individual capacity to eradicate enterococci biofilm- $0.2 \%$ CTR has been previously shown to eradicate $E$. faecalis ATCC 29212 biofilm after 30 seconds and using the same methodology (25). Previous studies also show that $0.2 \%$ CTR associated with other organic acids at clinical concentrations proved able to eradicate biofilms of E. faecalis ATCC 29212 after 1 minute (associated with citric acid) and even after just 30 seconds (associated with maleic acid) (9). Our results confirm that this association is indeed effective.

The association of LA with CHX exhibited less antibiofilm efficacy than LA alone. This reduced effect could be due to the fact that $\mathrm{CHX}$ at $2 \%$ is not capable in itself of eradicating biofilms of E. faecalis either at 30 seconds or at 1 minute (23). The results of the association could be considered intermediate, that is, bet-ween the results obtained when the two solutions are applied separately. From the chemical standpoint, LA, with its anionic nature, could have been neutralized by the bicationic molecule of CHX. However, it was considered interesting a priori to evaluate this combination because CHX has high substantivity, and its association with another anionic molecule, fluoride, has been seen to reduce cariogenic microorganisms in the long term (25) without altering the efficacy of the fluorides in the control of dental caries (26).

The different results obtained between the various single biofilms and the dual-species biofilm lead us to weigh the importance of testing irrigating solutions not only against $E$. faecalis ATCC 29212 but also against wild strains and above all against the polymicrobial biofilms that can be found in infected root canals. In this in vitro study, we were able to demonstrate that LA, aside from eliminating the smear layer (10) and improving the bond strength of Epiphany adhesive sealer (11) is capable of eradicating enterococci biofilm at a concentration of $20 \%$. The combination of lower concentrations of this acid with $0.2 \%$ CTR achieved eradication after 1 minute of exposure. Ex vivo studies are needed to verify these results in the complexity of the root canal system.

\section{References}

1. Figdor D, Sundqvist G. A big role for the very small--understanding the endodontic microbial flora. Aust Dent J. 2007;52:S38-51.

2. Sedgley CM, Lennan SL, Appelbe OK. Survival of Enterococcus faecalis in root canals ex vivo. Int Endod J. 2005;38:735-42.

3. Young GR, Parashos P, Messer HH. The principles of techniques for cleaning root canals. Aust Dent J. 2007;52:S52-63. 
4. Haapasalo M, Qian W, Portenier I, Waltimo T. Effects of dentin on the antimicrobial properties of endodontic medicaments. J Endod. 2007;33:917-25.

5. Zehnder M. Root canal irrigants. J Endod. 2006;32:389-98.

6. Pérez-Heredia M, Ferrer-Luque CM, González-Rodríguez MP. The effectiveness of different acid irrigating solutions in root canal cleaning after hand and rotary instrumentation. J Endod. 2006;32:993-7.

7. Ballal NV, Kandian S, Mala K, Bhat KS, Acharya S. Comparison of the efficacy of maleic acid and ethylenediaminetetraacetic acid in smear layer removal from instrumented human root canal: a scanning electron microscopic study. J Endod. 2009;35:1573-6.

8. Arias-Moliz MT, Ferrer-Luque CM, Espigares-García M, Baca P. Enterococcus faecalis biofilms eradication by root canal irrigants. J Endod. 2009;35:711-4.

9. Ferrer-Luque CM, Arias-Moliz MT, González-Rodríguez MP, Baca P. Antimicrobial activity of maleic acid and combinations of cetrimide with chelating agents against Enterococcus faecalis biofilm. J Endod. 2010;36:1673-5.

10. Ayad MF. Lactic acid root canal irrigation for dowel and core treatment: a pilot study. J Prosthet Dent. 2004;92:540-5.

11. Ayad MF, Farag AM, Garcia-Godoy F. Effect of lactic acid irrigant on shear bond strength of Epiphany adhesive sealer to human dentin surface. Oral Surg Oral Med Oral Pathol Oral Radiol Endod. 2010;109:e100-6.

12. Ayad MF, Bahannan SA, Rosenstiel SF. Morphological characteristics of the interface between resin composite and glass-ionomer cement to thin-walled roots: a microscopic investigation. Am J Dent. 2010;23:103-7.

13. Ayad MF, Bahannan SA, Rosenstiel SF. Influence of irrigant, dowel type, and root-reinforcing material on fracture resistance of thinwalled endodontically treated teeth. J Prosthodont. 2011;20:180-9.

14. Gadang VP, Hettiarachchy NS, Johnson MG, Owens C. Evaluation of antibacterial activity of whey protein isolate coating incorporated with nisin, grape seed extract, malic acid, and EDTA on a Turkey frankfurter system. J Food Sci. 2008;73:M389-94.

15. Ceri H, Olson ME, Stremick C, Read RR, Morck D, Buret A. The Calgary Biofilm Device: new technology for rapid determination of antibiotic susceptibilities of bacterial biofilms. J Clin Microbiol. 1999;37:1771-6.

16. Harrison JJ, Turner RJ, Ceri H. High-throughput metal susceptibility testing of microbial biofilms. BMC Microbiol. 2005;5:53.

17. Subramanian K, Mickel AK. Molecular analysis of persistent periradicular lesions and root ends reveals a diverse microbial profile. J Endod. 2009;35:950-7.

18. Ozbek SM, Ozbek A, Erdorgan AS. Analysis of Enterococcus faecalis in samples from Turkish patients with primary endodontic infections and failed endodontic treatment by real-time PCR SYBR green method. J Appl Oral Sci. 2009;17:370-4.

19. Lee Y, Han SH, Hong SH, Lee JK, Ji H, Kum KY. Antimicrobial efficacy of a polymeric chlorhexidine release device using in vitro model of Enterococcus faecalis dentinal tubule infection. J Endod. 2008;34:855-8.

20. Mejàre B. Streptococcus faecalis and Streptococcus faecium in infected dental root canals at filling and their susceptibility to azidocillin and some comparable antibiotics. Odontol Revy. 1975;26:193-204.

21. Chavez de Paz LE. Redefining the persistent infection in root canals: possible role of biofilm communities. J Endod. 2007;33:65262 .

22. Giardino L, Ambu E, Savoldi E, Rimondini R, Cassanelli C, Debbia EA. Comparative evaluation of antimicrobial efficacy of sodium hypochlorite, MTAD, and Tetraclean against Enterococcus faecalis biofilm. J Endod. 2007;33:852-5.

23. Arias-Moliz MT, Ferrer-Luque CM, González-Rodríguez MP, Valderrama MJ, Baca P. Eradication of Enterococcus faecalis biofilms by cetrimide and chlorhexidine. J Endod. 2010;36:87-90.

24. Baca P, Junco P, Arias-Moliz MT, González-Rodríguez MP, Ferrer-Luque CM. Residual and antimicrobial activity of final irrigation protocols on Enterococcus faecalis biofilm in dentin. J Endod. 2011;37:363-6.
25. Twetman S, Petersson LG. Efficacy of a chlorhexidine and a chlorhexidine-fluoride varnish mixture to decrease interdental levels of mutans streptococci. Caries Res. 1997;31:361-5.

26. Petersson LG, Magnusson K, Andersson H, Deierborg G, Twetman S. Effect of semi-annual applications of a chlorhexidine/fluoride varnish mixture on approximal caries incidence in schoolchildren. A three-year radiographic study. Eur J Oral Sci. 1998;106:623-7. 